\title{
Full-wave Numerical Modeling of an Infrared Free Electron Laser Using FDTD/PIC
}

\author{
Arya Fallahi * , Alireza Yahaghi ${ }^{* \dagger}$ and Franz X. Kärtner $* \ddagger \S$ \\ * Center for Free-Electron Laser Science, DESY, Notkestrasse 85, 22607 Hamburg, Germany \\ $\dagger$ Department of Communication and Electronics Engineering, Shiraz University, Jomhoori Eslami Blvd. Shiraz, Iran \\ $\ddagger$ Department of Physics, University of Hamburg, Jungiusstrasse 9, 20355 Hamburg, Germany \\ $\S$ The Hamburg Center for Ultrafast Imaging, Luruper Chaussee 149, 22761 Hamburg, Germany
}

\begin{abstract}
Free Electron Lasers (FELs) are a solution for providing intense THz and IR radiations with high brightness. Due to the low wall-plug efficiency of FEL facilities compared to lasers and other radiation sources, it is crucial and additionally very useful to develop complete simulation tools capable of accurately simulating a FEL process. The highly sophisticated dynamics involved in a FEL process was the main obstacle hindering the development of a general simulation tool for this problem. We present a numerical algorithm based on FDTD/PIC in a Lorentz boosted coordinate system which is able to completely simulate the FEL process. The developed software offers a suitable tool for the analysis of the FEL interaction without considering any of the usual approximations.
\end{abstract}

\section{INTRODUCTION}

$\mathbf{F}$ REE Electron Lasers (FELs) have recently garnered extensive attention due to their promising peculiarity of generating radiation in the whole electromagnetic spectrum. Among the different intervals of the spectrum, high power $\mathrm{THz}$ and X-ray generation is merely achieved by FEL based sources [1], [2]. Therefore, it is crucial and additionally very useful to develop sophisticated though complete simulation tools based on the main involved principles like Maxwell's equations and relativity. On one hand, such a tool can be used for the optimization of FEL output and on the other, novel schemes and ideas to achieve high quality radiations can always be verified by such a software. For example, the software presented in this study is mainly motivated by the authors to study the development of compact X-ray sources based on optical undulators.

The currently existing softwares are usually written to tackle special cases with particular approximations. Some examples of such approximations are 1D FEL theory, steady state, slow wave and forward wave approximation, wiggler-averaged electron motion and slices approximation. Many of these assumptions may lose their validity in certain regimes. The presented software aims the analysis of the FEL interaction without considering any of the above approximations. The developed tool apparently suffers from long computation times but offers a more accurate picture on the radiation process.

\section{Methodology}

In order to overcome the problem of multidimensionality, we exploit Lorentz boosted coordinate system and implement Finite Difference Time Domain (FDTD) method on the
Hemholtz equation governing the magnetic vector potential. The field solver is then coupled with a Particle in Cell (PIC) algorithm to simulate the particle motion in this frame. There are several important considerations to obtain reliable results converging to the real values. Examples are the method for electron bunch generation, particle pusher algorithm and computational mesh truncation, which need particular attention. For the sake of brevity, we do not explain these features in this contribution and discuss their precise implementation in another paper.

For solving the time domain Maxwell's equation, we use the well-known FDTD approach due to its inherent properties like explicit time update, zero DC fields, and zero self-force in the solution. Since we are dealing with field propagation in free space, solving Helmholtz equations is a better choice to achieve an efficient solver. It is well-known that the proper definition of the vector potential $(\boldsymbol{A})$ and scalar electric potential $(\varphi)$ recast Maxwell's equations for $\boldsymbol{E}, \boldsymbol{B}$ into two uncoupled equations, namely vector Helmholtz equation for $\boldsymbol{A}$ and scalar Helmholtz equation for $\varphi$ :

$$
\begin{aligned}
\nabla^{2} \boldsymbol{A}-\frac{1}{c^{2}} \frac{\partial^{2}}{\partial t^{2}} \boldsymbol{A} & =-\mu_{0} \boldsymbol{J} \\
\nabla^{2} \varphi-\frac{1}{c^{2}} \frac{\partial^{2} \varphi}{\partial t^{2}} & =-\frac{\rho}{\varepsilon_{0}}
\end{aligned}
$$

$c=1 / \sqrt{\mu_{0} \varepsilon_{0}}$ is the light velocity in vacuum. In the derivation of above equation the Lorentz gauge condition $\nabla \cdot \boldsymbol{A}=-\frac{1}{c} \frac{\partial \varphi}{\partial t}$ is used. The original $\boldsymbol{E}$ and $\boldsymbol{B}$ vectors are obtained from $\boldsymbol{A}$ and $\varphi$ as:

$$
\begin{gathered}
\boldsymbol{B}=\nabla \times \boldsymbol{A} \\
\boldsymbol{E}=-\frac{\partial}{\partial t} \boldsymbol{A}-\nabla \varphi
\end{gathered}
$$

To solve the above equations, without violating the charge conservation law, we solve the discretized form of (1) with electron currents (i.e. macro-particles) as the source in conjunction with the Lorentz gauge to evaluate the scalar potential. To obtain the fields $\boldsymbol{E}$ and $\boldsymbol{B}$ at the grid points, we use momentum conserving interpolation, which interpolates the field values to the grid points. The Lorentz gauge $\nabla \cdot \boldsymbol{A}=-\frac{1}{c} \frac{\partial \varphi}{\partial t}$ is solved similar to the Helmholtz equation through the discretization using the central finite differences. 
(a)

Bunch rest frame:

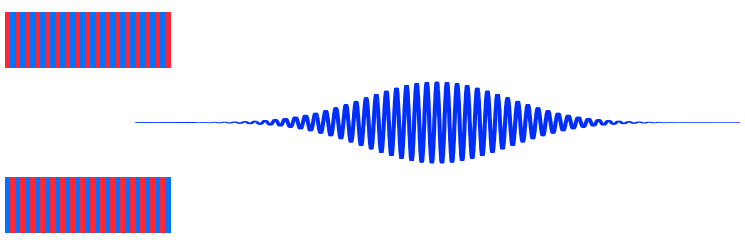

(b)

Fig. 1. Schematic illustration of the Lorentz boosting to transform the problem from the laboratory frame to the bunch rest frame.

To solve for the electron trajectories, particle in cell (PIC) method is utilized. The method takes the time domain data of the fields $\boldsymbol{E}$ and $\boldsymbol{B}$ and updates positions and momenta according to the relativistic equation of motion for macroparticles:

$$
\frac{\partial}{\partial t}(\gamma m \boldsymbol{v})=-e(\boldsymbol{E}+\boldsymbol{v} \times \boldsymbol{B}), \quad \text { and } \quad \frac{\partial \boldsymbol{r}}{\partial t}=\boldsymbol{v},
$$

where $\boldsymbol{r}$ and $\boldsymbol{v}$ are the position and velocity vectors of the macro-particles, $e, m$ and $\gamma$ are the electron charge, rest mass, and its Loretnz factor.

A novelty of the implemented technique is the solution of Maxwell's equations in the bunch rest frame. Based on the already developed and matured FEL theory, it can be demonstrated that coordinate transformation into the bunch rest frame causes all the involved parameters such as bunch length, undulator period, undulator length, and radiation wavelength converge from widely different length scales into values with same orders of magnitude. In Fig. 1, we present a schematic illustration of how transformation into the bunch rest frame offers this advantage.

\section{RESUlts}

Using the developed code, we have simulated an infrared FEL with the parameters tabulated in III and compared our FDTD/PIC solver with the standard FEL simulation code Genesis 1.3. The results for the radiated power with the traveled path along the undulator is shown in Fig. 2.

\section{CONCLUSION}

Using the FDTD algorithm to solve time domain Maxwell's equation combined with the PIC method to solve for the particle trajectories, a software is developed for the numerical modeling of a FEL process. An infra-red FEL is outlined and simulated using this software as well as Genesis 1.3.

\begin{tabular}{|c||c|}
\hline \multicolumn{1}{|c||}{ FEL parameter } & Value \\
\hline \hline Bunch type & Uniform cylinder \\
\hline Bunch size & $260 \times 260 \times 100) \mu \mathrm{m}$ \\
\hline Bunch charge & $51.4 \mathrm{MeV}$ \\
\hline Bunch energy & $88.5 \mathrm{~A}$ \\
\hline Bunch current & $0.01 \%$ \\
\hline Longitudinal momentum spread & 0.0 \\
\hline Transverse momentum spread & $3.0 \mathrm{~cm}$ \\
\hline Undulator period & $0.5 \mathrm{~T}$ \\
\hline Magnetic field & 1.4 \\
\hline Undulator parameter & $5 \mathrm{~m}$ \\
\hline Undulator length & $3 \mu \mathrm{m}$ \\
\hline Radiation wavelength & $8.7 \times 10$ \\
\hline Electron density & $20.0 \mathrm{~cm}$ \\
\hline Gain length (1D) & 0.006 \\
\hline FEL parameter & $39.7 \mu \mathrm{m}$ \\
\hline Cooperation length & 0.01 \\
\hline Initial bunching factor & \\
\hline
\end{tabular}

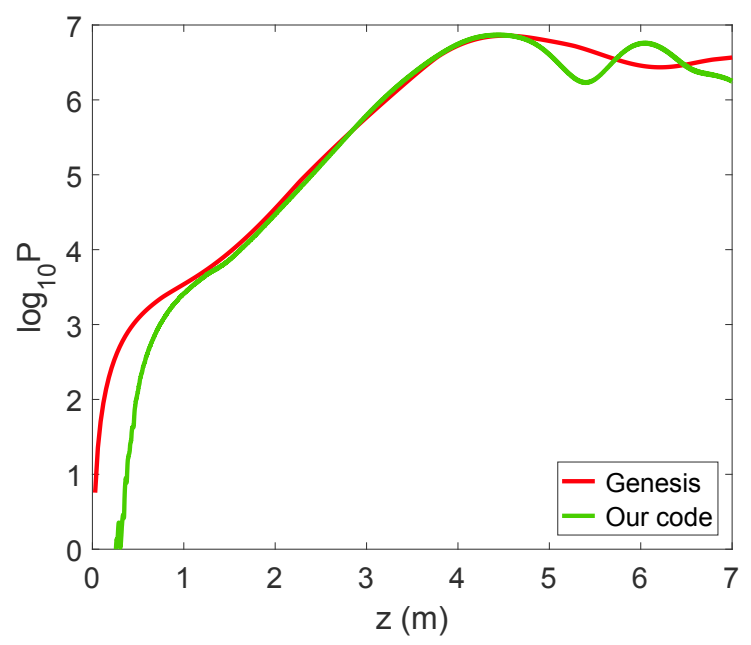

Fig. 2. Radiation power of an IR FEL in terms of the traveled path along the undulator.

The agreement between the results show the reliability of the results produced by both softwares.

\section{ACKNOWLEDGMENT}

This work was supported by the European Research Council under the European Union's Seventh Framework Programme (FP/2007-2013) / ERC Grant Agreement n. 609920, the Center for Free-Electron Laser Science at DESY and the excellence cluster "The Hamburg Centre for Ultrafast Imaging - Structure, Dynamics and Control of Matter at the Atomic Scale" of the Deutsche Forschungsgemeinschaft. A. Y. is acknowledging support by a George-Foster research fellowship from Alexander von Humboldt foundation.

\section{REFERENCES}

[1] J. H. Booske, R. J. Dobbs, C. D. Joye, C. L. Kory, G. R. Neil, G.-S. Park, J. Park, and R. J. Temkin, "Vacuum electronic high power terahertz sources," Terahertz Science and Technology, IEEE Transactions on, vol. 1, no. 1, pp. 54-75, 2011.

[2] C. Pellegrini, A. Marinelli, and S. Reiche, "The physics of x-ray freeelectron lasers," Reviews of Modern Physics, vol. 88, no. 1, p. 015006, 2016. 